\title{
Avaliação de Narrativas Orais em Crianças Falantes de Português Europeu (PE): Um Teste Piloto ${ }^{1}$
}

\author{
Stéphanie Dias Vaz*, Maria Lobo* \& Marisa Lousada** \\ *CLUNL NOVA FCSH \\ **Escola Superior de Saúde da Universidade de Aveiro/ CINTESIS.UA
}

\begin{abstract}
:
Although there are several referenced and validated international instruments for evaluating oral narratives, which can be used in populations with language disorders to help the diagnosis and to plan intervention, there is yet no such instrument for EP. In this work, we present a first version of an instrument for evaluating oral narrative productions for EP-speaking children, including the methodology used in the construction of the instrument, its structure, criteria for the analysis of productions, and procedures used in the validation of the instrument. In a second moment, we report the first results from a pilot study that indicate that there may be differences between tasks (telling or retelling), between types of stories and between age groups in what concerns the analysis of macrostructural parameters.
\end{abstract}

Keywords: Evaluation; Narratives; Oral production

Palavras-chave: Avaliação; Narrativas; Produção oral

\section{Introdução}

Para além do recurso a instrumentos formais de avaliação da linguagem que incidem sobre componentes específicas (fonológica, morfossintática, lexical), tais como a Avaliação da Linguagem Oral (ALO) (Sim-Sim, 2003), o Teste de Identificação de Competências Linguísticas (TICL) (Viana, 2004), o Teste de Linguagem ALPE (TL-ALPE) (Mendes et al. 2014), o Teste de Avaliação de Linguagem na Criança (TALC) (Sua Kay \& Tavares, 2006), o SinTACS (Vieira, 2011) a Grelha de Observação da Linguagem - Nível Escolar GOL-E (Sua Kay \& Santos, 2014), entre outros, a avaliação da linguagem oral pode ser complementada com avaliação da produção e da compreensão de segmentos discursivos maiores, incluindo a análise de discurso espontâneo ou a produção e compreensão de narrativas. Existem vários instrumentos dedicados à avaliação de competências narrativas para outras línguas, em particular para o inglês, mas, tanto quanto saibamos, não existe nenhum instrumento com objetivos semelhantes para o português.

No presente trabalho apresentamos um instrumento de avaliação de competências narrativas que se encontra em desenvolvimento para o português - o (RE)CONTO - e alguns resultados da aplicação de um estudo piloto desse instrumento.

O trabalho está estruturado da seguinte forma: na secção 2., discutimos a relevância de avaliar as competências de produção e compreensão de narrativas orais, caracterizando diferentes dimensões que podem ser consideradas nessa avaliação; na secção 3., fazemos uma revisão de trabalhos que consideraram o desenvolvimento de competências narrativas; na secção 4., apresentamos a versão piloto do instrumento; na secção 5., reportamos os resultados do teste piloto, seguindo-se as principais conclusões do estudo.

\footnotetext{
${ }^{1}$ Trabalho realizado no âmbito da Bolsa de Doutoramento KRUse atribuída a Stéphanie Vaz (PD/BD/131387/2017), financiada pela FCT, sob a orientação de Maria Lobo e Marisa Lousada.
} 


\section{Avaliação de narrativas}

\subsection{Relevância da avaliação de competências narrativas}

É habitual classificar-se os textos (orais e escritos) em categorias. Uma dessas categorias é a dos textos narrativos. Neste conjunto de textos, podem incluir-se relatos de acontecimentos pessoais, relatos de acontecimentos vividos por outros, narrativas ficcionais, entre outros. Na verdade, a caracterização de um texto como pertencendo a uma determinada categoria, neste caso a de texto narrativo, é mais complexa do que pode parecer. Adam $(1982,1987)$ defende que um texto é uma unidade que contém sequências prototípicas, segmentos textuais maiores do que a frase, que podem ser de vários tipos, incluindo sequências narrativas, descritivas, dialogais, explicativas e argumentativas. O conto, o romance, o relato ou a parábola serão textos em que predominam as sequências narrativas. Contudo, dentro de textos que classificamos como narrativos, podem encontrar-se outros tipos de sequências, incluindo sequências descritivas ou dialogais, por exemplo. Assim, apesar de um texto narrativo incluir predominantemente sequências narrativas e de a sua função ser a de contar uma sucessão de acontecimentos, na verdade ele pode incluir também sequências descritivas, dialogais e outras (Adam, 1982, 1987). É possível, por conseguinte, analisar de forma mais fina as características de diferentes textos narrativos, identificando as suas partes constitutivas e as sequências que integram.

A complexidade dos textos narrativos pode dever-se a diferentes fatores, que passam pela sua maior ou menor prototipicidade enquanto texto narrativo, pelas estruturas linguísticas usadas (léxico, estruturas sintáticas), pelo número e diversidade de situações relatadas, pelo número de personagens e relações que se estabelecem entre elas, entre outros fatores.

Embora haja múltiplas definições de narrativa, é relativamente consensual que uma sequência narrativa prototípica inclui diferentes momentos: um momento inicial, uma complicação, um conjunto de acontecimentos que visam repor o estado de equilíbrio, a resolução e a situação final ou desfecho (Todorov, 1973; Adam, 1982; 1987; Sousa, 2010). Um texto narrativo pode conter uma única sequência narrativa ou múltiplas sequências narrativas. Inclui sempre pelo menos um personagem e uma sucessão de acontecimentos situados espacial e temporalmente, ligados por relações de causalidade (Sousa, 2010).

Desde muito cedo, as crianças contactam com narrativas, ao ouvir contar histórias. As narrativas estão presentes em todas as sociedades, seja na modalidade escrita, seja através de narrativas de tradição oral (Labov \& Waletzky, 1997).

As narrativas são também textos muito trabalhados no primeiro ciclo de escolaridade (Pereira \& Azevedo, 2005), constituindo a produção de narrativas um objetivo transversal a todos os anos de escolaridade. Esta constatação reforça a ideia, defendida também por Gonçalves, Guerreiro \& Freitas (2011), de que as narrativas constituem bons instrumentos preditivos das competências linguísticas e comunicativas.

As competências de produção oral de narrativas compreendem a capacidade de produzir um texto estruturado que contém uma sequência de acontecimentos ligados por relações de causalidade e de ordenação temporal, e de manter mecanismos de coesão temporal e referencial, usando as construções linguísticas apropriadas. A compreensão de narrativas orais inclui a capacidade de entender a informação contida no texto, as relações de coesão, incluindo relações causais e temporais, bem como a capacidade de relacionar os eventos relatados com as experiências pessoais e retirar conclusões. A compreensão pode incluir diferentes processos, incluindo a compreensão literal, inferencial e crítica (Viana et al., 2010; Giasson, 2005; entre outros). Podemos, assim, incluir na competência narrativa, por um lado, a capacidade de produzir textos narrativos, isto é, textos cuja função é contar uma história, ainda que possam não incluir apenas sequências narrativas, e, por outro lado, a capacidade de compreender esses textos, incluindo a "lição" que podem transmitir - componente argumentativa que pode estar explícita ou implícita no texto (Adam, 1987).

A produção oral de narrativas mobiliza, assim, capacidades mais globais, relacionadas com a organização da informação no texto e suas partes constituintes (parâmetros macroestruturais), e capacidades mais 
específicas, relacionadas com as estruturas linguísticas usadas e a sua articulação (parâmetros microestruturais). Trata-se de uma tarefa linguística complexa, que requer domínio de estruturas linguísticas (como fórmulas de abertura e fecho, uso de conetores temporais e causais, uso de tempos verbais, entre outros), mas também de propriedades cognitivas (como memória de trabalho, capacidade de fazer inferências e de estabelecer relações temporais e causais) (Spinillo, 1993; Simões, 2000; Kaderavek \& Sulzby, 2000; Spinillo \& Brandão, 2001).

A produção oral de narrativas fornece, pois, informações acerca: i) dos conhecimentos sintáticos e semânticos, ii) do uso de mecanismos de construção de referência, de continuidade do tópico e de articulação de frases complexas, e iii) da capacidade de adaptação ao destinatário (Gonçalves, Guerreiro \& Freitas, 2011). Nas narrativas, a produção pressupõe não só a representação analítica dos acontecimentos, como também o emprego de marcas linguísticas que organizem esses acontecimentos (Rebelo, Marques \& Costa, 2000).

A avaliação destas competências permite completar o perfil linguístico de crianças avaliadas através de instrumentos mais formais, analisar competências gerais e específicas, e auxiliar os terapeutas da fala na avaliação, diagnóstico, planeamento e respetiva intervenção terapêutica. Para além disso, permite eventualmente antecipar dificuldades que se possam vir a manifestar na escrita de narrativas e na compreensão da leitura (Catts et al., 2015; Pinto et al., 2016). Assim, a avaliação de narrativas orais tem sido incluída a par de outros instrumentos de avaliação da linguagem, contemplando métodos de análise quantitativos e qualitativos (Justice et al., 2010; Petersen et al., 2008).

Até ao momento existe muita investigação sobre produções narrativas, havendo um número considerável de estudos baseados no conto Frog, Where Are You? (Mayer, 1969), um dos materiais universalmente utilizado com a finalidade de desencadear a produção de narrativas. Contudo, poucos são os instrumentos normalizados (Viana et al., 2017) com vista à avaliação e diagnóstico clínico nesta área da linguagem. Como referimos, existem vários instrumentos de avaliação de narrativas orais para outras línguas, em particular para o inglês, usados em contexto clínico, incluindo The Bus Story (Renfrew, 1969); Test of Narrative Language (TNL; TNL2) (Gillam \& Pearson, 2004; 2017); Expression, reception and recall of narrative instrument (ERRNI) (Bishop, 2004); Edmonton Narrative Norms Instrument (ENNI) (Schneider, Dubé \& Hayward, 2005); Diagnostic Evaluation of Language Variation - Norm Referenced Test (DELV-NR) (Seymour, Roeper \& Villiers, 2005); Multilingual Assessment Instrument for Narratives (MAIN) (Gagarina et al. 2015). Estes instrumentos distinguem-se quanto às faixas etárias alvo, quanto à forma como é induzida a narrativa e quanto aos parâmetros de análise considerados. No entanto, todos eles têm como procedimento comum uma tarefa de reconto, com ou sem ajudas materiais. Quando não há ajudas materiais, a criança apenas tem de escutar uma história narrada pelo avaliador e proceder ao seu reconto. Quando há materiais de suporte, são fornecidos cartões que ilustram a sequência de ações que constituem a narrativa. As faixas etárias em que as provas são aplicáveis centram-se entre os 3 e os 15 anos. A codificação e análise diferem de prova para prova; no entanto, incluem habitualmente a análise da macroestrutura (ou esquema narrativo) e a análise de elementos linguísticos, incluindo a análise dos recursos de coesão e a análise das hesitações.

Neste artigo, centrar-nos-emos nas competências de produção oral de narrativas. Na secção seguinte, descrevemos diferentes parâmetros que podem ser avaliados na produção oral de narrativas.

\subsection{Parâmetros de análise}

Como já referido, existem dois níveis que podem ser usados na análise da narrativa: uma análise de dimensão macroestrutural, e uma análise de dimensão microestrutural (Beaugrande \& Dressler, 1983; Graesser, Gernsbacher \& Goldman, 1997; Van Dijk, 1997). Estas duas análises representam duas áreas distintas, mas inter-relacionadas, subjacentes à competência narrativa (Liles et al., 1995; Pearson, 2002). A análise macroestrutural permite avaliar as capacidades de organização linguística mais gerais, enquanto a análise microestrutural permite registar e avaliar as capacidades de expressão linguística (morfossintáticas, lexicais) 
(Westby, 2005; Monteiro et al. 2016). Consideramos nesta secção esses dois níveis e destacamos em 2.2.3. as relações de tempo e causa.

\subsubsection{Análise macroestrutural}

A macroestrutura diz respeito a todos os componentes que possibilitam a organização global de sentido do texto e que são responsáveis pela sua significação. São estas componentes que tornam possível o planeamento, a compreensão, a memorização e a reprodução das ideias essenciais da narrativa. À macroestrutura associam-se, portanto, todos os elementos e mecanismos que visam manter a coerência do texto (Sautchuk, 2003). Da macroestrutura de um texto fazem parte as representações globais que permitem identificar as suas partes constituintes e o sentido do texto. Nas narrativas ficcionais, teremos geralmente um esquema mais específico com: uma situação inicial, que inclui uma localização temporal e espacial e a introdução de personagens, um evento perturbador, uma tentativa de resolução e um resultado dessa ação. Dependendo da maior ou menor complexidade das narrativas, pode haver uma só destas sequências ou várias sequências. O número de sequências narrativas do texto e a inclusão de outras sequências (descritivas ou dialogais, por exemplo) pode contribuir para a complexidade da estrutura do texto.

A análise macroestrutural considera a coerência da narrativa, incluindo a presença e a organização das partes constituintes da narrativa, com referência aos elementos relevantes da situação inicial, à sequência de eventos ligados por relações de causalidade, manutenção de personagem e tema, e relação entre eventos e desfecho (Spinillo \& Martins, 1997; Miles \& Chapman, 2002; Heilmann et al., 2010).

Diferentes instrumentos de avaliação de narrativas orais usam grelhas de codificação distintas para a análise da componente macroestrutural. A análise macroestrutural aplicada no instrumento MAIN, por exemplo, consta de uma adaptação amplamente divulgada na análise da Gramática da História introduzida por Stein \& Glenn (1979). A análise compreende os seguintes componentes macroestruturais: localização, evento inicial, finalidade, tentativa, desfecho e reação. O TNL inclui uma grelha que analisa o conteúdo da narrativa, verificando se a criança refere: i) os elementos do cenário - localização espaciotemporal e diferentes personagens; ii) os fatores que desencadeiam a sucessão de eventos; iii) cada um dos eventos constituintes da narrativa (ação e reação); e iv) o desfecho da narrativa.

\subsubsection{Análise microestrutural}

A dimensão microestrutural das narrativas engloba as propriedades lexicais, semânticas, sintáticas e morfológicas das frases que asseguram a coesão do texto. No nível microestrutural, os instrumentos costumam considerar medidas de complexidade das narrativas e a manutenção da coesão.

Segundo Halliday \& Hasan (1976), a coesão refere-se às relações de sentido estabelecidas através de elementos gramaticais e lexicais, que são responsáveis pela sua estruturação e que são cruciais para a sua interpretação. A microestrutura é, desta forma, responsável pela estruturação linguística do texto, e representa todo um sistema de mecanismos/elementos linguísticos que garantem a coerência textual e a coesão referencial, temporal, lexical (Sautchuk, 2003).

A análise microestrutural considera a sequencialidade e sentido da narrativa, além dos elementos de coesão temporal e referencial que regem a sequência estrutural, com base nos princípios morfossintáticos e semânticos.

Spinillo \& Martins (1997) concluem que a coesão, a coerência e a aquisição de um esquema narrativo estão intimamente relacionadas, e que o desenvolvimento da narrativa envolve todos esses aspetos de maneira integrada (Halliday \& Hasam, 1976; Justice et al., 2010).

A análise microestrutural permite, assim, analisar o desenvolvimento linguístico da criança e pode englobar várias componentes linguísticas: i) lexicais - incluindo, por exemplo, a diversidade lexical e o número de palavras diferentes; ii) morfológicas - incluindo, por exemplo, diversidade de morfemas flexionais usados e palavras gramaticais; iii) sintáticas e sintático-semânticas - incluindo medidas globais, como a extensão média 
e máxima de enunciado, ou medidas mais específicas, como a quantidade e diversidade de conectores usados, o número ou a proporção de frases complexas, os tipos de frases complexas produzidos, as marcas de coesão referencial, entre outros aspetos.

Os elementos de análise microestrutural considerados no MAIN (Gagarina et al. 2015) cobrem uma ampla gama de aspetos linguísticos, incluindo medidas gerais de extensão e diversidade lexical, aspetos da morfossintaxe e discurso. O TNL avalia a presença de marcação de relações temporais e causais entre eventos expressas através de conectores, o número de frases agramaticais, a presença de diálogo e a congruência dos eventos narrados, entre outros aspetos.

É sabido que as competências linguísticas da criança a nível microestrutural estão ainda em desenvolvimento em idade escolar, havendo diferenças entre crianças de diferentes faixas etárias. Também há diferenças nas narrativas de crianças com perturbações da linguagem e crianças com desenvolvimento típico no discurso narrativo, que se manifestam na extensão da história, no vocabulário, no desenvolvimento temático, na extensão média dos enunciados e no número de mecanismos/elementos linguísticos (Newman \& McGregor, 2006). A avaliação destes aspetos permite ao terapeuta da fala estabelecer um perfil linguístico da criança.

\subsubsection{Relações de tempo e causa}

Tal como refere Sousa (1996), a linguagem conserva marcas das operações que a constituem, atualizando um conjunto de marcadores que funcionam como sinais das operações mentais que estão na origem da produção de enunciados. Desta forma, para estudarmos as produções narrativas temos de ter em conta relações temporais e causais. Estes dois aspetos estão especificamente incluídos em alguns instrumentos de avaliação de narrativas, como é o caso do TNL.

Dado que a narrativa inclui uma situação inicial, uma sucessão de eventos desencadeados e um desfecho, tem de haver um número mínimo de eventos que possa assegurar essa sequência. Por outro lado, não é apenas o número de eventos que garante a sequencialidade, mas também as relações lógicas e temporais que se estabelecem entre os eventos, ao longo da narrativa. Essas relações são garantidas pela presença de conectores que marcam relações temporais, causais e consecutivas entre os eventos (Rosa, 2009).

O tempo tem um papel imprescindível na construção de uma narrativa, pois marca o período cronológico (tempo de um acontecimento), do início ao fim da história, ou seja, a história desenvolve-se no decorrer desse tempo. Também as relações temporais entre os eventos são fundamentais.

Vários autores assumem nos seus trabalhos que a narrativa remete sempre para um tempo e um espaço diferentes, disjuntos em relação à situação da enunciação (Batoréo, 1996; Silva, 2005; Sousa, 2007, e.o). Silva (2005) refere que a sequencialidade temporal e o predomínio de situações eventivas constituem as ideias-chave na expressão do tempo em sequências narrativas. Para este autor, "as propriedades macroestruturais estão relacionadas com o recurso a mecanismos linguísticos de expressão do tempo".

Segundo Oliveira (2003), todas as línguas possuem diferentes marcas responsáveis por localizar eventos ou estados no eixo temporal, no entanto, no caso do português europeu (PE), os falantes tendem a utilizar com maior frequência os tempos verbais (encontrando-se, nas narrativas, geralmente o passado - pretérito perfeito, pretérito imperfeito e pretérito mais-que-perfeito), embora os advérbios ou expressões adverbiais temporais e certas construções de tempo também possam desempenhar essa função. Posto isto, todas as produções narrativas retratam uma sucessão de acontecimentos que se passa num determinado tempo e lugar. A narração só existe na medida em que há ação, que é praticada pelos personagens; uma ação ocorre por uma determinada causa e desenrola-se envolvendo certas circunstâncias. Assim, é importante mencionar o modo como tudo aconteceu e as consequências provocadas pelos acontecimentos.

A causalidade é uma característica que diz respeito ao facto de as ações ao longo da narrativa se desenrolarem respeitando uma lógica, pautadas por relações de causa e efeito entre diferentes situações (Neves \& Oliveira, 2001; 
Sousa, 2010). Tudo isto é referido com apoio de marcadores linguísticos de causa e de consequência (expressões adverbiais, orações adverbiais, advérbios e/ou sintagmas preposicionais).

Costa, Vasconcelos e Sousa (2010) sugerem que uma história só faz sentido e é coerente quando existe uma linha temporal estruturada em que os episódios se ordenam, estabelecendo relações de simultaneidade, posterioridade e anterioridade. Também Silva (2005) indica que a sucessão de eventos introduz um critério de natureza temporal na definição de narrativa. E ainda Adam (1992) caracteriza as sequências narrativas como um conjunto de eventos que se sucedem no tempo ligados por relações de causalidade, incluindo diferentes momentos.

A análise das expressões que marcam relações de temporalidade e de causalidade pode, por conseguinte, ser relevante para determinar a competência narrativa da criança.

\section{Desenvolvimento de Competências Narrativas}

Nos últimos anos, de acordo com Monteiro et al. (2016), a investigação sobre aquisição da linguagem tem focado de forma significativa a organização da informação no processamento discursivo. A produção de uma narrativa coerente é uma tarefa linguística complexa que requer que o sujeito planeie e execute a verbalização atualizando múltiplas competências linguísticas (Cook \& O’Brien 2014). Na narrativa, a organização cronológica e causal de acontecimentos implica o uso de estruturas linguísticas para a expressão do tempo, a localização de entidades e acontecimentos, o que leva ao uso de marcadores de espaço; a referência a múltiplos participantes nos eventos obriga à utilização de formas de referência e de correferência complexas (Monteiro et al., 2016).

Vários estudos (Hudson, \& Shapiro, 1991; Silva \& Spinillo, 2000; Spinillo, 1993) mostram que as competências de produção de narrativas por parte das crianças aumentam de acordo com a idade/escolaridade, observando que as histórias se tornam cada vez mais complexas e elaboradas. De acordo com Gonçalves, Guerreiro \& Freitas (2011), o desenvolvimento discursivo emerge entre os dois e os quatro anos de idade e estabiliza no final da adolescência. As crianças aos cinco e seis anos apresentam conhecimento da estrutura da narrativa, com domínio satisfatório da relação temporal entre os eventos narrados, utilizando operadores narrativos e fazendo uso de relações espaciotemporais organizadas (Dadalto \& Goldfeld, 2009), a criança demonstra ser capaz de começar a narrar os «porquês» e os «modos» como os acontecimentos se desenrolam e estão já presentes nas suas narrativas sentimentos, intenções, emoções e pensamentos (Peterson \& McCabe, 1983; Umiker-Sebeok, 1979).

Para Silva (2001) a narrativa está presente na vida da criança desde o berço, daí que não seja de estranhar que desde muito cedo a estrutura básica da narrativa comece a emergir, pois, conforme diz a autora, o contar de uma história implica: ser capaz de processar e produzir enunciados que respeitem as leis da coesão e coerência; articular os episódios da história sujeitando-os às relações temporais e causais; e ainda, dominar a forma como se estruturam as narrativas, isto é, possuir a representação cognitiva do esquema de organização interna da narrativa (Sim-Sim, 1998).

A partir dos seis anos de idade, a criança apresenta uma maior flexibilidade dos esquemas de organização interna da narrativa e uma maior capacidade de seleção da informação relevante (Larrea, 1994).

Silva (2001) refere que o desenvolvimento cognitivo, social e linguístico da criança condiciona o desenvolvimento da competência narrativa, sendo possível estabelecer um padrão de desenvolvimento de acordo com a idade. A riqueza linguística do contexto em que a criança cresce, incluindo as experiências de literacia familiar e o contacto precoce com histórias, é um dos fatores mais importantes que condiciona o desenvolvimento da competência narrativa.

Bento \& Befi-Lopes (2010) assumem que o aumento da complexidade das narrativas e o aumento da idade desencadeiam um aumento do tipo de discurso intencional e diminuição do tipo de discurso descritivo. Assim, estas autoras afirmam que a capacidade de ordenação temporal já está desenvolvida aos sete anos e que as produções orais sofrem influência da idade e do tipo de narrativa fornecida. 
Diversos estudos revelam ainda que o desempenho narrativo das crianças mais novas melhora na presença de imagens ou sequências de imagens (Shapiro, \& Hudson, 1991; Zanotto, 2003; Cruz, 2011). Desta forma, a compreensão narrativa encontra-se condicionada pelo facto de a criança apoiar a sua compreensão em imagens, ou seja, esta está ainda dependente de um suporte concreto, figurativo e espacial (Veloso, 1999).

Outros autores têm dedicado os seus estudos à narrativa, comparando as capacidades e competências de crianças com e sem perturbações de linguagem (Gillam \& Johnston, 1992; Oetting \& Horohov, 1997; Kaderavek \& Sulzby, 2000; Scott \& Windsor, 2000; Conti-Ramsden, 2003). As competências narrativas nas crianças com perturbação de linguagem desenvolvem-se ao longo do tempo, mas estão abaixo das dos seus pares com desenvolvimento típico (Reuterskiöld et al., 2011).

Existem até ao momento vários estudos para o português, centrados em narrativas (escritas ou orais) que consideraram diferentes parâmetros de análise e, até mesmo, diferentes populações: crianças com dificuldades de aprendizagem, crianças surdas, crianças bilingues, entre outros.

Alguns desses trabalhos consideram o desenvolvimento de diferentes conectores e/ou expressões adverbiais temporais usados na produção de narrativas (escritas ou orais). Estes trabalhos mostram que há desenvolvimento na produção de diferentes conetores, associados a propriedades sintáticas e semânticas com diferentes graus de complexidade, e que esse parâmetro é relevante para avaliar o desenvolvimento linguístico da criança na produção da narrativa.

Costa (2010) estudou a produção escrita de conexões contrastivas por crianças e adolescentes, concluindo que a aquisição de nexos concessivos, semanticamente contíguos aos adversativos e aos condicionais, é tardia. A autora colocou a hipótese de que o carácter tardio da aquisição de conectores concessivos possa ser explicado pela complexidade estrutural destas unidades, representadas no léxico por configurações complexas de traços. Os resultados relatados em Costa (2010) foram comparados com a produção espontânea de conectores representativos de nexos contrastivos, causais e condicionais (cf. corpus de Santos, 2006).

De acordo com Costa et al. (2008) em estádios iniciais de aquisição é possível estabelecer uma escala de emergência que corresponderá à seguinte ordem de aquisição de conectores: e $>$ mas $>$ porque $>$ se. Esta escala aproxima-se bastante da escala apresentada por Diessel (2004) para o inglês: and $>$ because $>$ so $>$ but $>$ when $>$ if.

Borges (2015) estudou os efeitos do ensino explícito/desenvolvimento da consciência linguística na produção de narrativas escritas, mostrando que na fase de pré-teste os alunos usaram predominantemente a conjunção de coordenação 'e' e a conjunção subordinativa temporal 'quando' e que, depois de uma intervenção direcionada para os conectores, passaram a usar uma maior diversidade de conectores temporais, orações subordinadas adverbiais temporais e expressões adverbiais temporais.

Silva (2001) refere que a emergência dos conectores apresenta, tanto no escrito como no oral, um padrão de evolução bastante semelhante. Porém, apesar da similitude de evolução nas duas modalidades, regista-se um desfasamento nas idades. De acordo com a autora, o uso do marcador "e depois" nas crianças mais novas é o mais frequente, e usado quase em exclusivo. Já o grupo de crianças mais velhas apresenta uma maior diversidade de marcadores. O uso da conjunção subordinativa "quando", por exemplo, vai aumentando progressivamente, à medida que vai diminuindo o uso de marcadores pouco específicos. O leque de marcadores vai-se diversificando à medida que a idade progride (Silva, 2001).

Desta forma, sabe-se que há desenvolvimento no uso de diferentes tipos de conetores (Silva, 2001; Diessel, 2004; Costa, 2006, 2010; Costa el al., 2008, e.o), interessando verificar o uso de conetores de diferentes graus de complexidade que as crianças usam nas produções das suas narrativas.

Existe, assim, desenvolvimento das competências narrativas das crianças, quer no nível macroestrutural, quer no nível microestrutural. Embora ambas as dimensões estejam contempladas no instrumento que está em preparação, no estudo piloto que integra o presente trabalho, só iremos analisar as produções orais das crianças na dimensão macroestrutural. 


\section{Metodologia}

O presente trabalho está enquadrado num projeto que visa avaliar as produções narrativas em crianças falantes de PE entre os 6 e os 12 anos, e com ele pretende-se atingir os seguintes objetivos:

a) criar uma primeira versão de um instrumento de avaliação de produções narrativas orais para crianças com desenvolvimento típico de linguagem no PE;

b) definir padrões normativos nas produções orais das crianças, nas duas tarefas apresentadas (conto e reconto);

c) analisar e interpretar a presença de propriedades micro e macroestruturais nas produções narrativas das crianças.

Os participantes serão caracterizados segundo idade cronológica, escolaridade, região e género. A recolha de dados será efetuada de norte a sul do país e ilhas, de forma individual.

Neste artigo, descrevemos a primeira versão do instrumento de avaliação de narrativas que estamos a desenvolver, em particular a metodologia usada na sua validação e apresentamos um estudo piloto enquadrado nesse projeto. Com o estudo piloto, pretendeu-se testar o instrumento, a adequação das imagens e histórias ${ }^{2} \mathrm{e}$ das tarefas contempladas, a facilidade de aplicação e de análise dos resultados. Apresenta-se as características gerais do instrumento e os primeiros resultados da análise de parâmetros macroestruturais, contemplando diferentes variáveis - o tipo de história, a tarefa e a idade.

\subsection{Questões de Investigação}

Para conseguir atingir os objetivos acima mencionados, partimos das seguintes questões de investigação:

1. Que tarefa permite aferir de forma mais adequada o desenvolvimento linguístico nestas produções narrativas - conto ou reconto?

2. Que tipo de história é mais eficaz para um instrumento de avaliação de desenvolvimento da competência narrativa?

3. Que medidas são mais robustas/fiáveis para determinar o desenvolvimento linguístico e a qualidade das produções a nível macroestrutural?

Neste estudo piloto, centrar-nos-emos em particular na comparação entre as tarefas de conto e de reconto (questão 1) e na comparação entre as três histórias (questão 2) mas apenas quanto à análise do desenvolvimento de parâmetros macroestruturais. Colocamos, assim, as seguintes questões específicas:

a) Há diferenças entre a tarefa de conto e de reconto na análise de parâmetros macroestruturais?

b) Há diferenças entre as histórias na análise de parâmetros macroestruturais?

c) Há diferenças entre faixas etárias na análise de parâmetros macroestruturais?

\subsection{Hipóteses}

Após revisão da literatura, colocaram-se as seguintes hipóteses de investigação face ao nosso estudo piloto:

1. As crianças têm uma performance melhor nas produções narrativas quando em situações de reconto de histórias; (Bishop \& Edmunson, 1987; Zanotto, 2003; Nippold, et al., 2005; Cavalcante \& Mandrá, 2010; Silva, 2013; Andrade, 2017, Balciuniene \& Kornev 2016; entre outros).

2. O desempenho das crianças é condicionado pela complexidade da estrutura narrativa e das estruturas linguísticas, tendo desempenhos melhores em histórias com estruturas narrativas mais convencionais e estruturas linguísticas menos complexas.

\footnotetext{
${ }^{2}$ Optámos por usar o termo "história" para nos referirmos a cada um dos textos narrativos que usámos, que, como veremos, têm características macroestruturais e linguísticas distintas.
} 
3. Existem diferenças entre grupos etários nos parâmetros macroestruturais (Dadalto \& Goldfeld, 2009; Borges, 2015).

\subsection{Critérios de Inclusão}

Dada a importância de definir critérios de inclusão e exclusão adequados, tornou-se relevante estabelecer os seguintes critérios na seleção das crianças:

1. Não apresentar problemas cognitivos (esta informação é facultada pelos cuidadores e professores), nem de linguagem (todas as crianças serão avaliadas através da Grelha de Avaliação da Linguagem - nível escolar - GOL-E (Sua Kay \& Santos, 2014);

2. Apresentar inteligibilidade de fala que garanta ao avaliador o acesso ao conteúdo da narrativa oral (palavras ou enunciados);

3. Ser falante monolingue de PE;

4. Ter idade cronológica de 6 a 12 anos;

5. Frequentar o ano de escolaridade correspondente à idade.

\subsection{Participantes}

Este estudo piloto contou com uma amostra de 38 crianças, com idades compreendidas entre os 6 e os 12 anos, de ambos os sexos, residentes em Sintra, Lisboa e Torres Novas, todas elas falantes monolingues de PE.

\begin{tabular}{|c|c|c|}
\hline Grupo & N & Intervalo de idades \\
\hline 6 anos & 4 & $6 ; 0-6 ; 9$ \\
\hline 7 anos & 4 & $7 ; 5-7 ; 10$ \\
\hline 8 anos & 4 & $8 ; 3-8 ; 11,7$ \\
\hline 9 anos & 6 & $9 ; 0-9 ; 11$ \\
\hline 10 anos & 4 & $10 ; 1-10 ; 10$ \\
\hline 11 anos & 11 & $11 ; 1-11 ; 9$ \\
\hline 12 anos & 5 & $12 ; 0-12 ; 11$ \\
\hline TOTAL & $\mathbf{3 8}$ & \\
\hline
\end{tabular}

Quadro 1: Número total de participantes, divididos por faixa etária

\subsection{Instrumento}

Foi aplicada inicialmente um instrumento de avaliação da linguagem - a GOL-E - a todas as crianças, permitindo avaliar as capacidades linguísticas, ao nível de estruturas semânticas, morfossintáticas e fonológicas. A prova tem como objetivo a avaliação da linguagem oral e ajuda na identificação de problemas que possam interferir na aprendizagem, possibilitando a correlação com os nossos resultados.

Esta prova é a única aferida para o PE para as faixas etárias 6 - 10 anos, não existindo outra que contemple as restantes faixas etárias (entre os 10 e os 12 anos). Assim sendo, assume-se que uma criança com desempenho considerado normal para os 10 anos e sem diagnóstico de perturbação nesta faixa etária terá um desenvolvimento típico. Coube à investigadora e terapeuta da fala que desenvolveu este estudo identificar alguma situação atípica passível de levar à exclusão da criança do estudo.

No estudo final, as crianças serão ainda sujeitas à aplicação de uma prova de memória - RAVLT (Teste de Aprendizagem Auditivo-Verbal de Rey) (Rey, 1964) permitindo despistar problemas de memória verbal que 
eventualmente interfiram na tarefa de reconto. Este é um instrumento neuropsicológico muito utilizado na prática clínica para detetar problemas de memória, e considera-se eficaz na identificação dos princípios básicos relacionados com o processo de retenção de novas informações.

Estas duas provas (de linguagem e de memória) serão usadas complementarmente para correlacionar os resultados do nosso instrumento.

Nesta fase piloto, foram criadas três histórias, correspondendo todas elas a narrativas ficcionais: i) a Minhoca e o Pássaro, criada pela investigadora; ii) os Elefantes Espertalhões, adaptada de um livro infantil já publicado - Chibos Sabichões (González, 2016), e iii) a Fada Arco-Íris, traduzida e adaptada de um livro infantil espanhol - El hada del arco iris (Yebra, 2015).

Estas três histórias foram manipuladas para o trabalho experimental, apresentando diferentes graus de complexidade quanto às sequências de eventos e à complexidade linguística. Das três histórias, a primeira ('A Minhoca e o Pássaro') é a que apresenta uma estrutura narrativa mais convencional, com sequências predominantemente narrativas, e a que tem um menor grau de complexidade linguística, com menor número de orações complexas. A história dos Elefantes Espertalhões inicia-se com uma sequência descritiva e contém várias sequências dialogais. A história da Fada Arco-Íris é a que contém estruturas linguísticas mais complexas e relações mais complexas entre as personagens e sequências de eventos, contendo também algumas sequências descritivas.

Para cada história foram criadas: i) sequências de imagens ilustrativas (cf. Exemplo de história - Sequência de imagens 1) por uma designer profissional; ii) uma narrativa que acompanha as imagens; e iii) perguntas de compreensão.

As perguntas de compreensão permitem perceber se as crianças compreendem as histórias apresentadas e incluem perguntas que avaliam a compreensão literal, inferencial e crítica.

No nosso estudo, cada sequência de imagens foi testada como estímulo a conto ou como suporte a situação de reconto a diferentes grupos de crianças. Todas as histórias foram aplicadas nas duas tarefas propostas (conto e reconto), sendo que cada grupo teve acesso a duas histórias, uma por cada tarefa.

O nosso instrumento foi aplicado em suporte digital e em papel, de forma individual e com recurso a gravação áudio.

Abaixo, apresentamos um exemplo de sequência de uma das histórias.

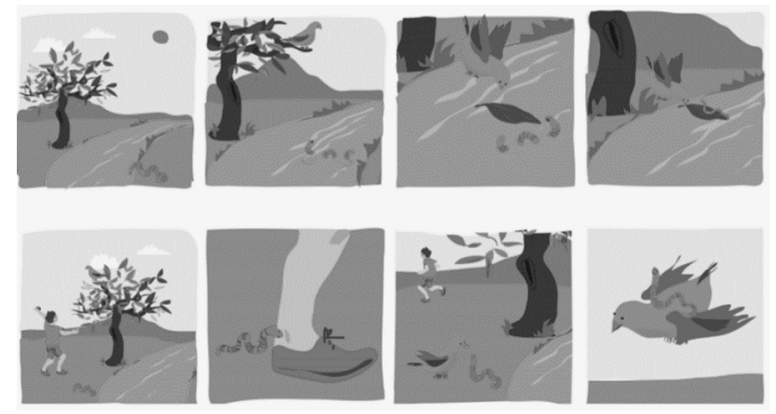

Sequência de imagens 1: História da minhoca e do pássaro

\subsection{Procedimentos}

Após a aplicação da prova de linguagem GOL_E, anteriormente referida, foi aplicado o nosso instrumento, tendo em conta os seguintes procedimentos para cada tarefa:

a) TAREFA DE CONTO:

1. Mostrar a sequência de imagens de uma história; 
2. Aplicar as perguntas de compreensão;

3. Pedir à criança que conte essa história.

b) TAREFA DE RECONTO:

1. Mostrar a sequência de imagens de outra história + ouvir a história;

2. Aplicar as perguntas de compreensão;

3. Pedir à criança que reconte essa história.

Todas as produções das crianças foram gravadas em áudio com consentimento prévio dos pais, e o projeto e materiais foram submetidos e aprovados pela comissão de ética da NOVA-FCSH e pelo Ministério de Educação.

\subsection{Critérios de Avaliação e Codificação}

As produções das crianças foram transcritas de acordo com o formato Chat (MacWhinney, 2000) e analisadas de acordo com uma grelha de critérios de desempenho, que contempla parâmetros macroestruturais e microestruturais. No entanto, neste estudo piloto só contemplamos parâmetros de avaliação macroestruturais, que incluem: abertura da narrativa; organização e progressão temática da narrativa; localização espacial e introdução de personagens; situação inicial; sequência de eventos; problema; e desfecho.

Como objetivos gerais do nosso projeto, serão ainda contemplados parâmetros microestruturais e eventuais desvios. Nos parâmetros microestruturais, serão incluídas: a) medidas de produtividade linguística da narrativa, referentes ao número total de palavras, número total de palavras diferentes e número de enunciados; b) medidas globais de complexidade linguística da narrativa, que incluem extensão média e máxima de enunciados em palavras; c) medidas de complexidade sintático-semântica, que incluem número e proporção de orações simples e complexas, tipo de frases complexas, número e diversidade de orações adverbiais e de conetores que expressam relações de tempo, causa e consequência. Serão ainda considerados eventuais desvios (problemas de concordância nominal ou verbal; de flexão regular e/ou irregular; uso inadequado de tempo e modo; de retoma pronominal; no uso de artigos...) e a manutenção de coesão referencial de entidades da narrativa.

\section{Resultados preliminares}

Neste estudo piloto, obtivemos um total de 38 respostas às perguntas de compreensão na tarefa de conto e 40 na tarefa de reconto, assim como 38 produções orais na tarefa de conto e 40 produções orais na tarefa de reconto (cf. Quadro 2). 


\begin{tabular}{|c|c|c|}
\hline & Conto & Reconto \\
\hline H1 - Minhoca & 16 & 13 \\
\hline H2 - Elefantes & 11 & 13 \\
\hline H3 - Fada & 11 & 14 \\
\hline TOTAL & $\mathbf{3 8}$ & $\mathbf{4 0}$ \\
\hline
\end{tabular}

Quadro 2: Número de produções orais e respetivos questionários de compreensão por cada história, nas duas tarefas apresentadas, conto e reconto

Quanto aos resultados observados nas perguntas de compreensão, em geral, há menor dificuldade nas perguntas de compreensão na tarefa de reconto; as crianças não apresentam respostas incompletas, mas as crianças mais novas responderam muitas vezes com "outras respostas", o que mostra a necessidade de reformulação das perguntas de compreensão numa versão posterior.

Relativamente aos parâmetros macroestruturais, apresentaremos, de seguida um gráfico explicativo que permite comparar os grupos etários, as tarefas e as diferentes histórias.

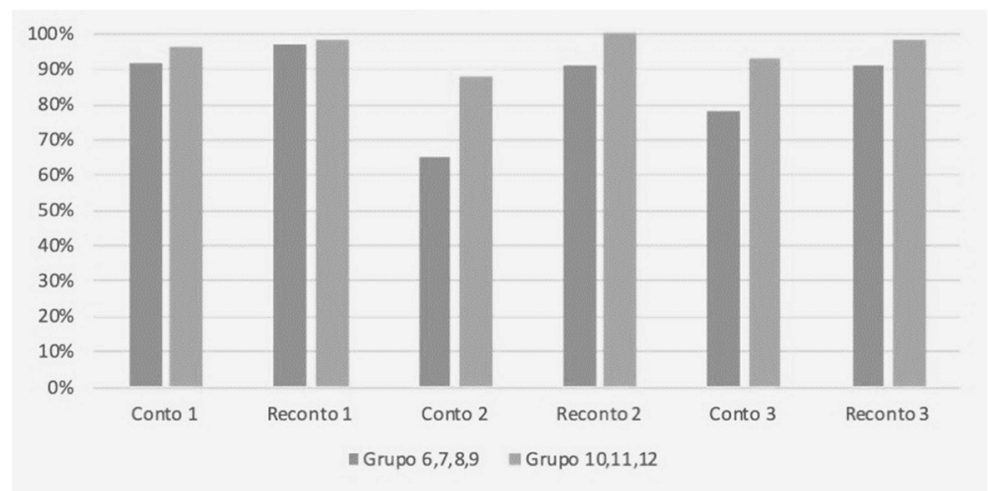

Gráfico 1: \% de acerto dos parâmetros macroestruturais por grupos etários

Perante os resultados, verificamos que existe uma taxa de acerto maior na tarefa de reconto em todas as histórias. Verificamos também que as crianças mais velhas obtêm resultados superiores aos das crianças mais novas.

A história 1- A minhoca e o pássaro, a que tem sequências predominantemente narrativas, com uma sucessão de eventos claramente relacionados por relações de causa-efeito, apresenta maior percentagem de acerto em todas as faixas etárias, e a história 2 - Elefantes espertalhões, que integra uma sequência descritiva, várias sequências dialogais e uma sucessão de episódios repetitivos, apresenta uma menor percentagem de acerto, com diferenças entre os dois grupos (6-9 vs. 10-12) mais assinaláveis sobretudo na tarefa de conto.

Concluindo, com estes resultados preliminares parecem existir:

i. possíveis diferenças entre a tarefa de conto e de reconto, sendo esta última a que induz maior facilidade na interpretação das imagens;

ii. possíveis diferenças entre as três histórias apresentadas;

iii. menor dificuldade nas perguntas de compreensão na tarefa de reconto; 
iv. diferenças entre a tarefa de conto e de reconto, nos parâmetros macroestruturais da narrativa, tais como os obtidos por vários autores (Bishop \& Edmunson, 1987; Zanotto, 2003; Nippold, et al., 2005; Cavalcante \& Mandrá, 2010; Silva, 2013; Borges, 2015; Balciuniene \& Kornev 2016; Andrade, 2017).

\section{Conclusões}

Neste estudo, apresentámos a primeira versão de um instrumento de avaliação de narrativas orais e alguns resultados da aplicação de um teste piloto deste instrumento. Globalmente, este estudo permitiu-nos verificar que as imagens e as histórias funcionaram de forma adequada na elicitação de narrativas, que uma das histórias - a da Minhoca e do Pássaro - é menos vulnerável ao efeito de tarefa, funcionando bem quer na tarefa de conto, quer na de reconto inclusivamente com crianças de faixas etárias mais baixas, sendo as outras duas histórias mais afetadas pela tarefa, com melhores resultados na tarefa de reconto e com desempenhos melhores nas faixas etárias mais velhas. O estudo piloto permitiu ainda afinar as questões de compreensão e identificar o momento mais adequado para a sua aplicação, bem como melhorar os critérios usados na análise das produções narrativas.

Esta foi uma etapa essencial no desenvolvimento do instrumento. Contudo, para que o instrumento possa ser usado em crianças falantes do PE como prova normalizada de avaliação de narrativas orais, capaz de identificar problemas de linguagem e de servir de apoio na intervenção, em trabalho futuro, será necessário obter dados sobre a sua adequação e facilidade de aplicação junto de um painel de peritos (vários profissionais das áreas da Terapia da Fala, Didática e Linguística). Será também necessário validar o instrumento, testando uma amostra maior e geograficamente mais diversificada, e avaliar as suas propriedades psicométricas (Denman et al. 2017; Souza et al. 2017). Pretende-se ainda aplicar uma prova de memória - RAVLT (Teste de Aprendizagem Auditivo-Verbal de Rey) a todas as crianças, garantindo um percentil adequado face à sua faixa etária, e permitindo identificar possíveis correlações entre memória e desempenho na tarefa de reconto. Finalmente, será necessário realizar uma análise mais robusta abrangendo tanto os parâmetros macroestruturais da narrativa como os microestruturais, de forma a que a versão final do instrumento seja capaz de estabelecer adequadamente perfis de desenvolvimento.

\section{Agradecimentos}

Gostaríamos de agradecer de forma especial a todos os pais que, de boa vontade e disponibilidade, aceitaram que os seus filhos pudessem participar neste estudo piloto; ao centro de estudos - SpotKids por permitir a recolha de dados nas suas instalações, e ao Eduardo pela preciosa ajuda com as transcrições. Agradecemos também aos dois revisores anónimos os seus comentários, que permitiram melhorar a versão final deste artigo.

\section{Referências:}

Adam. J. M. (1982) The Macro-Structure of the Conventional Narrative. Poetics Today. 3 (4) : 135-168.

Adam J.M. (1987) Types de séquences élémentaires. In: Pratiques : linguistique, littérature, didactique. Les types de textes. 56 : 54-79.

Adam, J. M. (1992) Textes: Types et Prototypes. Paris: Hachette.

Adam, J. M. (2011) The narrative sequence : history of a concept and a research area. In ler Colloque International organisé par le Réseau Romand de Narratologie (RRN). Redefinition de la sequence dans la narratologie postclassique. Université de Fribourg, 20-21 mai.

Andrade, J. (2017) A Importância do reconto de histórias no desenvolvimento cognitivo de crianças dos 3 anos. Dissertação de Mestrado em Ciências da Educação: Educação Especial Domínio Cognitivo e Motor e Intervenção Precoce. Porto: Universidade Fernando Pessoa. 
Balciuniene, I. \& Kornev, A. (2016) Doing new things with language: Narrative language in SLI preschoolers. Eesti Rakenduslingvistika Uhingu Aastaraamat 12, pp. 25-42.

Batoréo, H. (1996) Expressão do espaço no português europeu: contributo psicolinguístico para o estudo da linguagem e cognição. Lisboa: Tese de Doutoramento, Lisboa: FLUL.

Beaugrande, R. A. \& Dressler, W. U. (1983) Introduction to text linguistics. London: Longman.

Bento A., Befi-Lopes D. (2010) Organização e narração de histórias por escolares em desenvolvimento típico de linguagem. Pró-Fono Revista de Atualização Científica. 22 (4), pp. 503-508.

Bishop, D. V. M., \& Edmundson, A. (1987) Language impaired 4- year-olds: Transient from persistent impairment. Journal of Speech and Hearing Disorders, 52.

Bishop, D. V. M. (2004) Expression, Reception, and Recall of Narrative Instrument - ERRNI. London, UK: Harcourt Assessment.

Borges, I. (2015) Expressão de tempo e construção de narrativa. Dissertação de Mestrado em Desenvolvimento e Perturbações da Linguagem na Criança. Faculdade de Ciências Sociais e Humanas e Escola Superior de Saúde do Instituto Politécnico de Setúbal.

Catts HW, et al. (2015) Early prediction of reading comprehension within the simple view framework. Reading and Writing. 28 (9), pp. 1407-25.

Cavalcante, P. \& Mandrá P. (2010) Oral narratives of children with typical language development. Pró-Fono Revista de Atualização Científica. 22 (4), pp. 391-396.

Conti-Ramsden, G. (2003) Processing and linguistic markers in young children with specific language impairment (SLI). Journal of Speech Language and Hearing Research, Rockville, 46 (5), pp. 1029-1037.

Cook, A. \& O'Brien, E. (2014) Knowledge Activation, Integration, and Validation during Narrative Text Comprehension. Discourse Processes: A Multidisciplinary Journal, 51 (1-2), pp. 26-49.

Costa, A. L. (2006) Complexidade Estrutural de Conectores Concessivos. Textos Seleccionados do XXII Encontro da Associação Portuguesa de Linguística. Lisboa: APL.

Costa, A. L. el al. (2008) Efeitos de modelização no input: o caso da aquisição de conectores. Textos Seleccionados. XXIII Encontro Nacional da Associação Portuguesa de Linguística, Lisboa, APL.

Costa, A. L. (2010) Estruturas contrastivas: desenvolvimento do conhecimento explícito e da competência de escrita. Tese de doutoramento, Linguística (Linguística Educacional), Universidade de Lisboa, Faculdade de Letras.

Costa, A., Vasconcelos, S. \& Sousa, V. (2010) Muitas ideias, um mar de palavras. Propostas para o ensino da escrita. Lisboa: FCG.

Cruz, M. (2011) As histórias como recurso para o desenvolvimento da linguagem oral. Relatório da Prática de Ensino Supervisionada realizado para obtenção do grau de Mestre em Educação Pré-escolar. Faro: Universidade do Algarve, Escola Superior de Educação e Comunicação.

Dadalto, E. \& Goldfield, M. (2009) Características comuns à narrativa oral de crianças na pré-alfabetização. Revista CEFAC, Jan-Mar; 11(1), pp. 42-49.

Denman, D., et al. (2017) Psychometric Properties of Language Assessments for Children Aged 4-12 Years: A Systematic Review. Frontiers in Psychology, 8: 1515.

Diessel, H. (2004) The Acquisition of Complex Sentences. Cambridge: Cambridge U. P.

Freitas, A. (2006) O desenvolvimento narrativo na infância. Tese de Mestrado não publicada, Instituto de Educação e Psicologia, Universidade do Minho, Braga.

Gagarina, N. et al. (2015) Assessment of narrative abilities in bilingual children. In S. Armon-Lotem, J. de Jong \& N. Meir (eds.) Methods for assessing multilingual children: Disentangling bilingualism from language impairment. Bristol, UK: Multilingual Matters.

Gillam, R. \& Johnston, J. (1992) Spoken and written language relationships in language/learning- impairment and normally achieving school-age children. Journal of Speech Language and Hearing Research, Rockville, 35 (6), pp. 1303-1315. 
Gillam, R. B., \& Pearson, N. A. (2004) Test of Narrative Language - TNL. Austin, TX: ProEd.

Gillam, R. B., \& Pearson, N. A. (2017) Test of Narrative Language -TNL2. Austin, TX: 2ªd.

Giasson, J. (2000) A compreensão na leitura. Porto: Edições Asa.

Giasson, J. (2005) La lecture: de la théorie à la pratique. Bruxelas: De Boeck \& Larcier.

Gonçalves, F., Guerreiro P. \& Freitas, M. (2011) O conhecimento da língua; Percursos de Desenvolvimento. Lisboa: DGIDC, Ministério da Educação.

González, O. (2016) Chibos Sabichões. Kalandraka. Portugal.

Graesser, A. C., Gernsbacher, M. A. \& Goldman, S. (1997) Cognition. In: Van Dijk, T. (Ed.), Discourse: A multidisciplinary introduction, London: Sage, pp. 292-319.

Halliday, M. A. K., \& Hasan, R. (1976) Cohesion in English. English Language Series, London: Longman.

Heilmann, J. et al. (2010) Properties of the narrative scoring scheme using narrative retells in young school-age children. American Journal of Speech-Language Pathology, 19, pp. 154-166.

Hudson, J., \& Shapiro, L. (1991) From knowing to telling: The development of children's scripts, stories, and personal narratives. In A. McCabe \& C. Peterson (Eds.), Developing narrative structure. New Jersey: Lawrence Erlbaum Ass. pp. 89-135.

Justice L. M., et al. (2010) A scalable tool for assessing children's language abilities within a narrative context: the NAP (Narrative Assessment Protocol). Early Child Res Q. 25 (2), pp. 218-34.

Kaderavek, J. N., \& Sulzby, E. (2000) Narrative production by children with and without specific language impairment: oral narratives and emergent readings. Journal of Speech, Language, and Hearing Research, 43, pp. 34-49.

Labov W. \& Waletzky. J. (1997) Narrative Analysis: Oral Versions of Personal Experience. Journal of Narrative and Life History. 7 (1-4), pp. 3-38.

Larrea, M. G. (1994) La Comprensión de Cuentos en los Niños: Un enfoque Cognitivo y Sociocultural. Madrid: Siglo XXI de España Editores, S. A.

Liles, B. et al. (1995) Measurement of narrative discourse ability in children with language disorders. Journal of Speech and Hearing Research, 38, pp. 415-425.

MacWhinney, B (2000). The CHILDES Project: Tools for Analyzing Talk. $3^{\text {rd }}$ Edition. Mahwah, NJ: Lawrence Erlbaum Associates.

Mayer, M. (1969) Frog, Where Are You? New York: Penguin.

McKeough, A. (2000) Building on the oral tradition: How story composition and comprehension develop. In J. W. Astington (Ed.), Minds in the making: Essays in honor of David R. Olson. Malden: Blackwell Publishing. pp. 98-114.

Mendes, A. et al. (2014) Teste de linguagem (TL-ALPE). Aveiro: Edubox

Miles, S., \& Chapman, R. S. (2002) Narrative content as described by individuals with Down syndrome and typically developing children. Journal of Speech, Language, and Hearing Research, 45, pp. 175-189.

Monteiro, P. et al. (2016) Parâmetros para análise de narrativas orais em crianças com e sem perturbação da linguagem expressiva. Revista da Associação Portuguesa de Linguística, 1, pp. 621-650.

Neves, D., \& Oliveira, V. (2001) Sobre o Texto: Contributos teóricos para práticas textuais. Lisboa: Asa.

Newman, R.M., \& McGregor, K.K. (2006) Teachers and laypersons discern quality differences in narratives produced by children with or without SLI. Journal of Speech, Language, and Hearing Research, 49, pp. 1022-1036.

Nippold, M., el al. (2005) Conversational versus expository discourse: A study of syntactic development in children, adolescents, and adults. Journal of Speech, Language, and Hearing Research, 48, pp. 1048-1064.

Oetting, J. \& Horohov, J. (1997) Past-tense marking by children with and without specific language impairment. Journal of Speech Language and Hearing Research, Rockville, 40 (1), pp. 62-74. 
Oliveira, F. (2003) Tempo e Aspecto. In Maria Helena Mira Mateus et al. Gramática da Língua Portuguesa. $6^{\mathrm{a}}$ edição. Lisboa: Caminho, pp. 127-178.

Pearson, B. Z. (2002) Narrative competence among monolingual and bilingual school children in Miami. In Oller, K. \& Eilers, R. (Eds.), Language and literacy in bilingual children. Clevedon: Multilingual Matters.

Pereira, L. Á., \& Azevedo, F. (2005) Como abordar... A Escrita no $1{ }^{\circ}$ Ciclo do Ensino Básico. Porto: Areal Editores.

Petersen, D. et al. (2008) Emerging Procedures in Narrative Assessment: The Index of Narrative Complexity. Topics in Language Disorders, 28, pp. 115-130.

Peterson, C., \& McCabe, A. (1983) Developing narrative structure: Three ways of looking at a narrative. New York: Plenum.

Pinto G., et al. (2016) Development in narrative competences from oral to written stories in five-to seven-yearold children. Early Childhood Research Quarterly, 36, pp. 1-10.

Rebelo, D., Marques, M. J. \& Costa, M., L. (2000) Fundamentos da Didática da Língua Portuguesa. Lisboa: Universidade Aberta.

Renfrew, C. (1969) The Bus Story Test: a test of narrative speech. Bicester: Speechmark Publishing Ltd.

Reuterskiöld, C., et al. (2011) Narrative skills in Swedish children with language impairment. Journal of Communication Disorders, 44 (6), pp. 733 - 744.

Rosa, C. (2009) A Estrutura da Narrativa na Escrita dos Alunos Surdos. Dissertação de mestrado em Ciências da Educação. Instituto Politécnico De Lisboa Escola Superior De Educação De Lisboa.

Santos, A. L. (2006) Minimal Answers. Ellipsis, syntax and discourse in the acquisition of European Portuguese. Dissertação de Doutoramento. Universidade de Lisboa.

Sautchuk, I. (2003) A produção dialógica do texto escrito: um diálogo entre escritor e leitor interno. São Paulo: Martins Fontes.

Schneider, P., Dubé, R.V. \& Hayward, D. (2005) Edmonton narrative norming instrument - ENNI. Edmonton: University of Alberta.

Scott, C. \& Windsor, J. (2000) General language performance measures in spoken and written narrative and expository discourse of school-age children with language learning disabilities. Journal of Speech Language and Hearing Research, Rockville, 43 (2), pp. 324-339.

Seymour, H.N., Roeper, T.W. \& De Villiers, J. (2005) Diagnostic evaluation of language variation (DELV) Norm-referenced. San Antonio: Pearson.

Schneider, P., Dubé, R. \& Hayward, D. (2005) Edmonton Narrative Norms Instrument (ENNI). Retrieved from University of Alberta Faculty of Rehabilitation Medicine.

Shapiro, L. R., \& Hudson, J. A. (1991) Tell me a make-believe story: coherence and cohesion on young children's picture-elicited narratives. Developmental Psychology, 27 (6), pp. 960-974.

Shiro, M. (2003) Genre and evaluation in narrative development. Journal of Child Language, 30: 165-195.

Silva, I. (2013) O Reconto Oral e a autoria na Educação Pré-Escolar - Um estudo de caso. Dissertação de mestrado. Universidade de Aveiro. Departamento de Educação.

Silva, M. E. L., \& Spinillo, A. G. (2000) A influência de diferentes situações de produção na escrita de histórias. Psicologia: Reflexão e Crítica, 13 (3), pp. 337-350.

Silva, M. E. (2001) O desenvolvimento da competência narrativa: Uma análise de narrativas orais e escritas produzidas por sujeitos de 6, 7, 9, 11 e 14 anos. Dissertação de mestrado em Linguística - Teoria do Texto. FCSH-UNL.

Silva, P. (2005) O Tempo no Texto - Contributos para o estudo da expressão do tempo em sequências textuais. Universidade Aberta.

Sim-Sim, I. (1998) Desenvolvimento da Linguagem. Lisboa: Universidade Aberta.

Sim-Sim, I. (2003) Avaliação da Linguagem Oral: Um contributo para o conhecimento do desenvolvimento linguístico das crianças portuguesas. Lisboa: Fundação Calouste Gulbenkian. 
Simões, V. (2000) Histórias infantis e aquisição de escrita. São Paulo em Perspectiva, 14 (1), pp. 22-28.

Sousa, O. (1996) Construindo histórias. Lisboa: Estampa.

Sousa, O. (2000) Adverbiais temporais na construção da narrativa. In Gärtner, E., Hundt, C. \& Schönberger, A. (eds). Estudos de gramática portuguesa (I). Frankfurt am Main, Teo Ferrer de Mesquita, pp. 63-79.

Sousa, O. (2007) Tempo e Aspecto - O Imperfeito num Corpus de Aquisição. Lisboa: Edições Colibri e Instituto Politécnico de Lisboa.

Sousa. O. (2010) Emergência e desenvolvimento da relação de causalidade em narrativas de crianças. Filologia e Linguística Portuguesa, 12 (1), pp. 91-104.

Souza. A, Alexandre, N. \& Guirardello, E. (2017) Psychometric properties in instruments evaluation of reliability and validity. Epidemiologia e Serviços de Saúde, Brasília, 26 (3), pp. 649-659.

Spinillo, A. G. (1993) Era uma vez... E foram felizes para sempre: esquema narrativo e variações experimentais. Temas de psicologia, 1, pp. 67-87.

Spinillo, A. \& Martins, R. (1997) Uma análise da produção de histórias coerentes por crianças. Psicologia: Reflexão e Crítica., Porto Alegre, 10 (2), pp. 219-248.

Spinillo, A. \& Brandão, A. C. (2001) Produção e compreensão de textos em uma perspectiva de desenvolvimento. Estudos de Psicologia, 6 (1), pp. 51-62.

Stein, N, \& Glenn, G. (1979) An analysis of story comprehension in elementary school children. In R. Freedle (Ed.), New directions in discourse processing, Norwood, NJ: Ablex. (2), pp. 32-120

Sua-Kay, E. \& Tavares, M. (2006) TALC - Teste de avaliação da linguagem na criança. Lisboa: Oficina Didáctica.

Sua Kay, E. \& Santos, M. (2014) Grelha de Observação da Linguagem - Nível Escolar (GOL-E). $2^{\mathrm{a}}$ ed. Escola Superior de Saúde do Alcoitão.

Todorov, T. (1973) Qu'est-ce que le structuralisme?: Poétique. Paris: Éditions du Seuil.

Todorov, T. (2008) As estruturas narrativas. 5. São Paulo: Perspetiva.

Umiker-Sebeok, D. J. (1979) Preschool Children's Intraconversational Narratives. Journal of Child Language, $6(1)$, pp. 91-110.

Van Dijk, T. A. (1997) Discourse as structure and process. London: Sage

Veloso, T. (1999) A Compreensão de narrativas e a produção do discurso. Tese de Mestrado não publicada, Instituto de Estudos da Criança, Universidade do Minho, Braga.

Viana, F. (2004) TICL - Teste de identificação de competências linguísticas. Vila Nova de Gaia: Edipsico.

Viana, F. et al. (2010a) O ensino da compreensão leitora. Da teoria à prática pedagógica. Coimbra: Almedina.

Viana, F. et al. (2010b) Aprender a Compreender torna mais fácil o saber. Coimbra: Almedina.

Vieira, S. (2011) A test for sentence development in European Portuguese (STSDPT). Lisboa: Universidade de Lisboa. Tese de doutoramento.

Viana, F. et al. (2017) Instrumentos de avaliação da linguagem: uma perspetiva global. In Maria João Freitas \& Ana Lúcia Santos (eds.), Aquisição de língua materna e não materna: Questões gerais e dados do português, Berlin: Language Science Press, pp. 333-357.

Westby, C. E. (2005) Assessing and remediating text comprehension problems. In Catts, H.W. \& Kamhi, A. G. (eds) (2nd), Language and Reading Disabilities. Boston, MA; Pearson Education, Inc, pp. 157-232.

Yebra, A. (2015) El hada del arco iris. ChiquiCuentos. $11^{\text {a }}$ edicion, Editorial Bruño. Espanha.

Zanotto, M. (2003) Recontar Histórias - Atividade é importante para a formação das crianças pré-escolares. Revista do Professor, Porto Alegre, 74, pp. 5-9. 